\title{
¿POR QUÉ ELIGEN LOS ESTUDIANTES UNIVERSITARIOS LAS TITULACIONES DE TURISMO? ANÁLISIS DE LOS FACTORES DE INTERÉS Y EXPECTATIVAS DE EMPLEO PARA EL ALUMNADO DE LA UNIVERSITAT DE VALÈNCIA
}

\author{
María Caballer-Tarazona \\ Universitat de València \\ https://orcid.org/0000-0001-9242-0464 \\ Rubén José Cuñat Giménez. \\ Universitat de València \\ https://orcid.org/0000-0002-4424-1932 \\ Rosario Martínez Verdú \\ Universitat de València \\ https://orcid.org/0000-0002-9971-3531 \\ Cristina Pardo-García \\ Universitat de València \\ https://orcid.org/0000-0002-8068-3256
}

\section{RESUMEN}

Las expectativas del alumnado que determinan la elección de una titulación en Turismo condicionan sus conductas durante y después de sus estudios. Para identificar estas expectativas se realizó una encuesta al alumnado de Turismo de primer curso. Un análisis factorial nos ha permitido identificar los factores de mayor peso en la elección: Internacionalización, Expectativas de empleo, Emprendimiento, Importancia del sector e Interés por la titulación. Finalmente, se ha completado el estudio analizando sus expectativas sobre las salidas profesionales vinculadas al ámbito turístico.

Palabras clave: Motivaciones estudios universitarios en turismo; expectativas laborales en el sector turístico; análisis factorial; Grado en Turismo.

Fecha de recepción: 4 de junio de 2018

Fecha de aceptación: 28 de febrero de 2019

Departamento de Economía Aplicada, Universitat de València. Facultat d'Economia. Avda. Tarongers, s/n.46022 VALENCIA (España). E-mail: maria.caballer@uv.es, ruben.cunat@uv.es, rosario.martinez@uv.es, cristina.pardo-garcia@uv.es 


\title{
Why do university students choose tourism degrees? Interests analysis and employ- ment expectations for students from Universitat de València
}

\begin{abstract}
Interests that drive students to choose a Tourism degree will condition their behaviour in their studies and later on. In order to identify such interests we carried out a survey addressed to the first year Tourism students. A factorial analysis was conducted finding the main factors in the degree choice: Internationalization, Job expectations, Entrepreneurship, Sector importance and Interest for the degree. Finally, we completed the research identifying the expectations about their professional future in the tourism field.
\end{abstract}

Keywords: Motivations in tourism university studies; job expectations in tourism sector; factorial analysis; Degree in Tourism.

\section{INTRODUCCIÓN}

En España el Turismo es uno de los pilares básicos de la economía nacional, generando en el año 2016 ingresos por valor total de 125.529 millones de euros, calculados a través de la demanda final, lo que supone un 6,6\% más que el año anterior, siguiendo una senda alcista desde el año 2013 tras la crisis. Por otra parte, representa el 11,2\% del PIB español, según la Cuenta Satélite del Turismo España (CSTE) 2010-2016 publicada por el Instituto Nacional de Estadística (INE). También en términos de empleo tiene un peso relevante, puesto que en el año 2016 el Sector dio empleo a 2,56 millones de personas, cifra que equivale al $13 \%$ de la ocupación total, según la CSTE.

Todos estos datos dan cuenta de la solidez y el peso de este sector, convirtiéndose así en un elemento estratégico de la economía española. Por otra parte, España es actualmente la tercera economía que mayor número de visitas de turistas recibe, tras Francia y Estados Unidos, logrando en 2017 un registro de 81,4 millones de visitantes, lo que supone un crecimiento del 8,6\% con respecto al año anterior, según los datos definitivos de la encuesta Frontur publicada por el INE. Según la misma fuente, los turistas extranjeros que visitaron España gastaron un total de 86.823 millones de euros, con un aumento del $12,2 \%$ respecto al año anterior.

Un sector con tanto peso en la economía demanda unos recursos humanos motivados, fidelizados y bien formados, máxime considerando que la atención al cliente está presente en la mayor parte de las actividades que lo componen. Sin embargo, el análisis de las necesidades formativas en el sector es complejo, puesto que la formación es diversa. En cada uno de estos subsectores (hostelería, restauración, agencias de viaje, transportes, ocio, etc.) se demandan perfiles formativos determinados con necesidades de especialización y profesionalización diferentes (Lillo, 2009). A toda esta complejidad hay que añadir la estacionalidad, que genera empleo precario, temporal y diferencias laborales entre el segmento primario (alta cualificación y remuneración, empleo estable) y el 
secundario (baja cualificación y remuneración, alta rotación y temporalidad) (Marrero, Rodríguez y Ramos, 2016). Todo esto supone un importante reto para la formación de los trabajadores dentro del sistema educativo español en general y del universitario en particular.

En este sentido, la formación universitaria favorece a los grupos directivos y profesionales del sector, puesto que estos demandan un título universitario para el ejercicio profesional. Sin embargo, no sólo son necesarios los contenidos formativos para cubrir la demanda que se exige, sino que es necesario que las personas que cursan estudios universitarios de Turismo dispongan de la suficiente motivación para responder a las demandas del cliente.

La motivación, que podría estar relacionada con un aspecto vocacional, no siempre se produce, fundamentalmente porque la elección de carrera es un proceso complejo en el cual inciden diferentes factores, tanto personales como contextuales. Además, se lleva a cabo en una edad donde la juventud se encuentra inmerso en un proceso de definición de su identidad, apareciendo en escena las expectativas familiares y la variada información que transcurre en su entorno. Desde esta situación, la universidad debe ser capaz de recoger las expectativas del alumnado e incluirlas en su proceso formativo, con el objetivo de conseguir motivarle y orientarle hacia las salidas profesionales que mejor respondan a sus objetivos y perfil.

El objetivo general de este trabajo se centra en identificar qué expectativas o intereses llevan al alumnado a la elección de estudios universitarios en Turismo. Para ello, se propone:

- Sintetizar las diferentes expectativas que han llevado a la elección del Grado en grupos homogéneos o factores y establecer una jerarquía.

- Identificar las diferencias entre las expectativas del alumnado de primer curso de Grado en Turismo y Doble Grado en Turismo-Administración de Empresas (TADE) de la Universitat de València que han podido influir en la elección del grado.

- Identificar las expectativas de empleo del alumnado respecto a las principales salidas profesionales conectadas con el Grado de Turismo.

La orientación basada en las expectativas del alumnado constituye un enfoque poco estudiado en la literatura especializada. Por otra parte, no se encuentran trabajos que aborden este tema centrándose en los estudios universitarios en Turismo, por lo que nuestra aportación contribuye a generar conocimiento sobre el tema y sirve de base para la toma de decisiones en materia de planes de estudio.

El trabajo se estructura de la siguiente manera. En el apartado segundo realizaremos un breve repaso sobre la evolución de la formación universitaria en Turismo, desde la aparición de las directrices que permitían desarrollar los planes de estudio de las Diplomaturas de Turismo, hasta el actual Grado y su implementación en la Universitat de València. A continuación, se identifican y repasan los distintos enfoques que determinan la elección de una carrera universitaria. En la sección cuatro se propone la metodología que nos permitirá abordar los objetivos propuestos. Seguidamente, se analizan y discuten los principales resultados obtenidos. Finalizaremos con las conclusiones más relevantes del estudio. 


\section{LA FORMACIÓN UNIVERSITARIA EN TURISMO EN ESPAÑA}

La formación en turismo en España no fue regulada hasta la aparición del Decreto de 7 de septiembre de 1963, existiendo hasta entonces un vacío legislativo que hacía imposible la existencia de estudios orientados a capacitar a profesionales para puestos de responsabilidad en empresas del sector turístico; dándose esta formación en las propias empresas (ANECA, 2004). Es en este año cuando comienza a impartirse el título de "Técnico de Empresas Turísticas" con una duración de tres años (dos años de contenido común y un último de especialidad). Unos años más tarde, en 1980, y como respuesta a las demandas del sector, aparece el nuevo título de "Técnico en Empresas y Actividades turísticas", donde se reconocían los cambios que se habían producido en el campo turístico y la necesidad de adecuar la formación a la realidad y contribuir así a la mejora de la imagen del país.

En lo que respecta a la educación universitaria, las primeras Escuelas oficiales en España aparecen en 1989, donde se imparten estudios por un periodo de tres años, incorporando materias generales y específicas, así como idiomas, cuyo objetivo era capacitar al alumnado que deseaba incorporarse al mercado laboral turístico (Araújo y Fraiz, 2013). En 1996 aparecieron las directrices que permitían desarrollar los planes de estudio de las Diplomaturas en Turismo, con planes de estudio similares en todas las universidades en las que se impartía, con ligeras diferencias según la especialidad ofertada por cada una (Mariscal, 2003).

Posteriormente, a mediados de la década del 2000, aparecieron los programas de Postgrado en Turismo, que actualmente se han transformado en Másteres universitarios, a los que podían acceder los diplomados en turismo. Estos programas de postgrado se crearon totalmente integrados en el Espacio Europeo de Educación Superior, donde las materias se miden en créditos ECTS (considera la carga total de trabajo del estudiante, no solo en el aula sino también en aspectos de preparación y estudio de la asignatura). Como complemento a los estudios superiores en Turismo el Real Decreto 56/2005 reguló también el Doctorado en Turismo, que suponía un paso importante hacia la especialización e investigación.

Finalmente, la Ley Orgánica 4/2007 de 12 de abril, estableció tres ciclos en las enseñanzas universitarias: Grado, Máster y Doctorado. La principal diferencia respecto al marco legislativo que regulaba las anteriores titulaciones estriba en que desaparece el catálogo único de titulaciones establecido por el Gobierno, y cada universidad dispone de autonomía para crear y proponer, en función de normas establecidas, contenidos que se adapten a sus necesidades, recogiendo las fortalezas y oportunidades que se deriven del entorno en el que se encuentran ubicadas.

En el libro Blanco del Grado de Turismo (ANECA, 2004) se establecen los ejes básicos (Figura 1) de las materias por las que se apuesta en el Título: restauración, alojamiento, gestión pública y privada, recursos turísticos, diseño de productos turísticos e investigación turística, entre otros; considerando una orientación tanto al sector público como privado.

En España la duración mínima de los grados se estableció en 240 créditos, aunque posteriormente el Real Decreto 43/2015 de 2 de febrero estableció una nueva ordenación 
de las enseñanzas universitarias oficiales que permite a las universidades ofertar grados a partir de 180 créditos; teniendo los Másteres una duración entre 60 y 120 créditos.

En la Universitat de València el plan de estudios del título de Graduado/a en Turismo entró en vigor en el curso 2010-2011, quedando extinguido a partir de ese momento el plan correspondiente al título oficial de diplomado/a en Turismo. El Grado actual se distribuye en 240 créditos ECTS y tiene como objetivo proporcionar una formación que responda a las necesidades de dirección y gestión de empresas turísticas, de planificación de los destinos turísticos y de actividades relacionadas con el turismo urbano y de eventos, negocios y congresos (Universitat de València, 2009). Por lo que respecta a los estudios de TADE, el doble grado comenzó a impartirse en la Universitat de València en el curso 2014-15. Estos estudios no constituyen un título propio sino que permiten simultanear ambas titulaciones y obtener los grados en Turismo y en Administración y Dirección de Empresas. En este trabajo consideramos tanto la titulación de Turismo como la doble titulación de TADE, teniendo en cuenta que nuestro principal interés es analizar los estudios universitarios en Turismo, incluidos en ambas titulaciones.

\section{Figura 1 \\ PRIMERA APROXIMACIÓN CONCEPTUAL AL GRADO DE TURISMO}

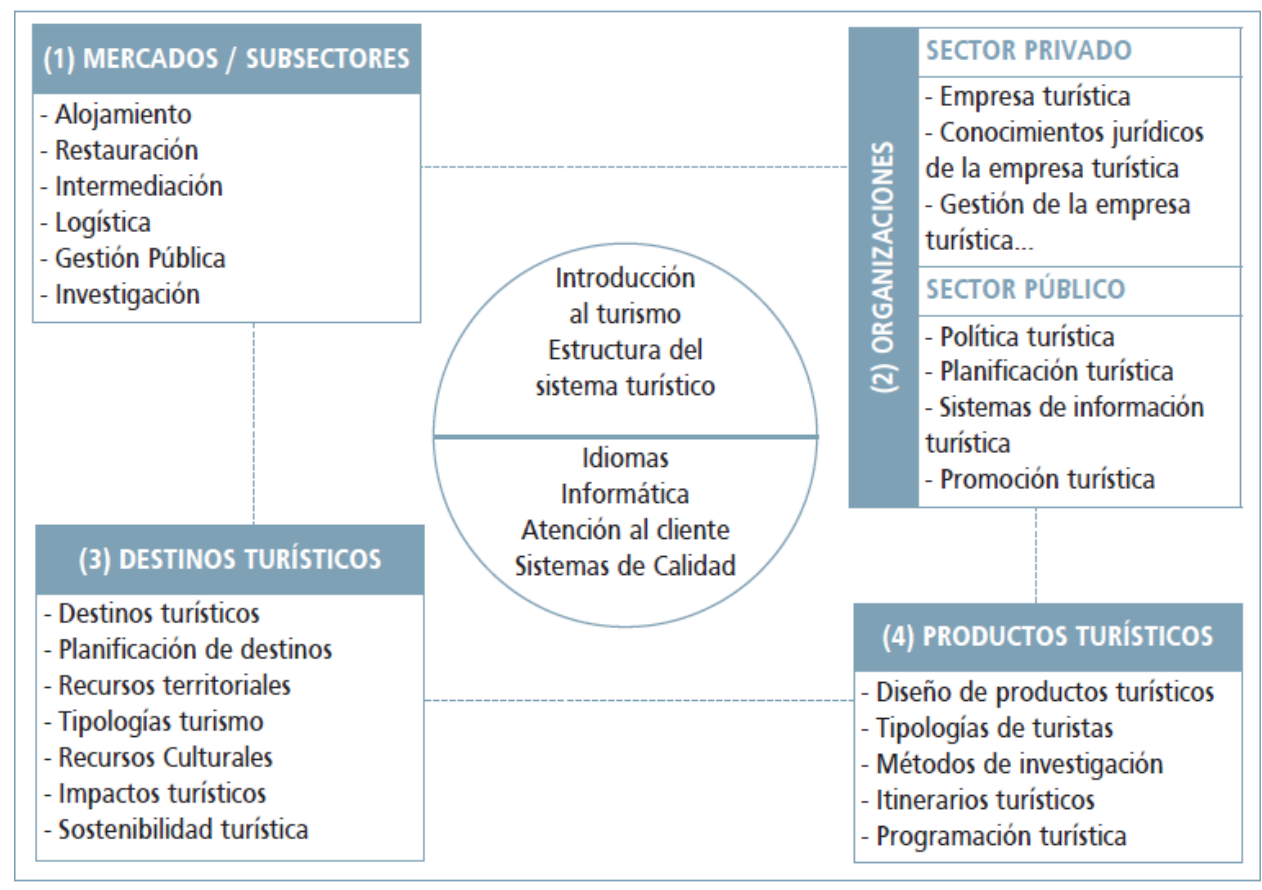

Fuente: Libro Blanco del Grado de Turismo, ANECA (2004). 
La adecuación de los Planes de Estudios a la realidad del mercado laboral favorece la inclusión del alumnado en el mismo. En los últimos años la naturaleza del trabajo ha ido experimentando muchos cambios, tanto en aspectos tecnológicos como organizativos. Estos cambios no han sido ajenos al Sector Turístico, obligando a las instituciones educativas a adaptar sus planes de estudios a las necesidades del entorno.

Dentro de este contexto entra en juego el concepto de "empleabilidad" que cada vez más se vincula con las instituciones de educación superior, no sólo por la capacitación profesional y acción formadora de los centros formativos, sino también porque este concepto es un indicador de calidad para las universidades, si lo entendemos desde el punto de vista de tasas de inserción laboral. En este sentido, el mercado laboral reclama trabajadores con un elevado nivel de capacidades y habilidades, con capacidad para solucionar problemas y adaptarse rápidamente al entorno (Suárez, 2014). Sin embargo, es importante considerar que la universidad debe mantenerse a una distancia óptima de las demandas del mercado laboral, puesto que si bien debe cubrir las necesidades que este demanda, también debe ofrecer una formación flexible y adaptable a los titulados; puesto que el rol actual de las universidades no puede ser exclusivamente docente, sino que debe cumplir una triple misión: docencia, investigación y transferencia a la sociedad (Oliván, 2014).

En esta combinación de necesidades que debe satisfacer la Universidad es necesario que el alumnado adquiera competencias amplias, que le permitan incorporar sus conocimientos a distintas situaciones y contextos, para poder desempeñar tareas altamente cualificadas (Hofstadt y Gómez, 2006).

Según el Libro Blanco del Grado de Turismo (ANECA, 2004) la actividad turística se mueve en un mercado laboral complejo, donde concurren diferentes competencias profesionales y amplias necesidades formativas. Se necesita orientar la formación hacia una realidad que obliga a disponer de un ciclo generalista (grado) donde el estudiante adquiere los conocimientos, competencias y habilidades necesarias para ejercer las múltiples tareas que se precisan, y una formación de postgrado que permite al alumnado profundizar y especializarse en aquellos ámbitos profesionales que le resulten de interés dentro del Turismo.

En el proyecto de la OCDE "Definition and Selection of Competencies" (DeSeCo) se dice que una competencia es más que conocimiento y habilidades, e implica la capacidad para poder cumplir con demandas complejas, movilizando recursos psicosociales (incluyendo habilidades y actitudes) en un contexto particular. Siguiendo la misma línea, Martínez y Saudela (2005) indican que la competencia siempre se refiere a destrezas, capacidades internas, habilidades, dominio, práctica y experiencia conseguida por el estudiante, por lo que precisa de una enseñanza centrada en este y de una evaluación que considere no solo los conocimientos adquiridos, sino también el saber hacer procedimental.

El libro Blanco del Grado de Turismo (ANECA, 2004) establece 22 competencias transversales agrupadas en tres categorías: instrumentales, personales y sistémicas; considerando que diez de ellas están integradas dentro de la lista final de competencias específicas del titulado de turismo, nueve deben desarrollarse de forma interdisciplinar entre las diversas competencias específicas y tres se incorporan de forma autónoma como competencias específicas de la formación. 


\section{FACTORES QUE INTERVIENEN EN LA ELECCIÓN DE UNA CARRERA UNIVERSITARIA}

La correcta elección de una carrera universitaria es un aspecto de sumo interés tanto a nivel social como económico, puesto que además de tener una repercusión directa sobre la planificación de los estudios en los centros universitarios, tiene importantes implicaciones en la implementación de políticas públicas dirigidas a la distribución de recursos, teniendo también efecto sobre la adecuación entre la demanda del mercado laboral y la oferta futura de profesionales que las diferentes titulaciones universitarias van a proporcionar.

Podemos encontrar en la literatura sobre el tema diferentes enfoques relacionados con los predictores en la elección de una carrera universitaria; algunos de estos ponen énfasis en las habilidades que el individuo considera que tiene respecto a diferentes temas, otros incluyen aspectos demográficos, económicos, sociales, de personalidad, familiares e incluso relacionados con la orientación política del individuo (Porter y Umbach, 2006). Todo esto hace que sea complejo encontrar un nexo común que agrupe todos estos enfoques y que contribuya a generar un cuerpo único en el estudio de los motivos que llevan a una persona a elegir una determinada carrera universitaria.

No obstante, lo que sí parece común en todos los enfoques es el papel que tiene la motivación en la elección de los estudios universitarios. En este sentido, al margen de consideraciones de orden técnico como la nota de corte para poder cursar una determinada carrera, el estudio de los mecanismos motivacionales que conllevan la elección pueden explicar las diferentes conductas que contribuyen tanto a la elección de los estudios como al rendimiento del alumnado en la carrera elegida.

Para Santrock (2002; pag. 432) la motivación es "el conjunto de razones por las que las personas se comportan de la forma que lo hacen". Este autor desarrolla tres perspectivas diferentes respecto a la motivación: la conductivista, la humanista y la cognitiva. La conductivista pone énfasis en la importancia de las recompensas en la motivación, la humanista en la capacidad del individuo para desarrollarse y elegir su destino y la cognitiva pone énfasis en lo que el individuo considera que puede controlar de forma efectiva en función de las capacidades que tiene.

En el enfoque conductual la motivación de los individuos es extrínseca, es decir, se basan en el deseo de conseguir alguna recompensa material en el futuro (prestigio, empleo bien remunerado, etc.). La motivación les impulsa a elegir aquellas carreras con mayor prestigio académico (Gámez y Marrero, 2003) para conseguir sus objetivos. Morris y Maisto (2001) justificaron que los estudiantes muestran no solo necesidades académicas, sino también económicas, interesándose por los beneficios que pueden obtener de ella a largo plazo.

Por otra parte, la perspectiva humanista descansa sobre la motivación intrínseca, siendo una de las teorías más conocidas en la explicación del comportamiento del individuo la jerarquía de las necesidades propuesta por Abraham Maslow en 1951. Maslow atribuye a las necesidades humanas una jerarquía, donde unas son prioritarias y solo se puede ascender a necesidades de orden superior cuando las inferiores están cubiertas. Dentro de este enfoque también encontramos la teoría de las necesidades de McClelland (1989), que 
señala los motivos sociales asociados al logro, afiliación y poder que tienen gran influencia en la vida de los estudiantes. En este sentido, Gámez y Marrero (2003) identifican como metas de logro la posibilidad de que la carrera permita conocer el resultado del esfuerzo, poder emprender un camino y llegar hasta el final o superar un reto importante. Respecto a las metas de afiliación: ayudar a los demás, hacer nuevas relaciones o amigos, sentirse parte de un grupo, etc.; y como metas de poder: poder influir sobre otras personas, ser un líder, conseguir una posición de poder, etc.

Finalmente, el enfoque cognoscitivo también pone énfasis en la motivación intrínseca, aunque orientada hacia la curiosidad, la satisfacción por aprender y el sentimiento de triunfo. En este sentido el individuo elegiría una carrera universitaria por su deseo de aprender una profesión que le motiva, condicionado por la percepción que tiene sobre el éxito futuro en esa área profesional, la capacidad que considera que tiene para controlar de forma efectiva el entorno en el que se presupone se moverá y las expectativas percibidas en la consecución de metas futuras.

La interacción de estos enfoques afecta a la motivación del estudiante, en positivo o negativo, por lo que se puede asociar con resultados académicos negativos si las expectativas no responden a las percepciones finales del alumnado.

En otro orden de cosas, existen estudios que indican que los individuos, a la hora de seleccionar una carrera universitaria, se guían por estereotipos ocupacionales que derivan de determinadas carreras de su entorno social, y que un individuo elige sus estudios en función del grado de satisfacción que piensa le va a producir (Holland, 1985). Otros autores como Alonso y Lobato (2004) argumentan que el 20\% del alumnado se matricula en titulaciones universitarias no deseadas y provocan abandonos académicos, con el consiguiente desencanto personal y la consecuente repercusión económica.

También la elección de una carrera universitaria se ha enfocado hacia aspectos psicológicos y no psicológicos del individuo (López y Norzagaray, 2016). Dentro de los enfoques no psicológicos se analizan aspectos sociológicos y económicos (Chacón, 2004), mientras que la perspectiva psicológica analiza la elección como un acontecimiento puntual que se produce en un determinado momento del tiempo (Lofquist y Dawis, 1969).

Por su parte, autores como Lent, Brown y Hackett (1994) clasifican los factores que intervienen en la elección de carrera en tres: personales, contextuales y experienciales. Los factores personales son aquellos inherentes al individuo, como la personalidad, interés, aptitud o género, entre otras. Los contextuales están relacionados con el entorno cercano del individuo como familiares, amigos, conocidos, etc. y también al nivel socioeconómico y características de la carrera. Mientras que los factores experienciales se refieren a los servicios y clases de orientación vocacional, experiencia laboral o prácticas.

Guàrdia et al., 2012, indican que el alumnado dentro del proceso de elección se ve influido por factores sociales e individuales. Los primeros son aquellos vinculados con la percepción de la universidad y la tradición de la titulación (es un grado de prestigio, es un sector importante en la economía del país, es un sector creciente), mientras que los factores individuales se relacionan con los propios intereses personales del estudiante, que pueden ser de carácter académico o no (me gusta la carrera, es una oportunidad para trabajar en el extranjero, podré aprender idiomas, puedo contribuir al crecimiento 
de mi país, tengo un negocio o trabajo ya en el sector, etc.). No obstante, estos mismos autores indican que también hay que incluir como condicionante de la elección los aspectos contextuales derivados del valor de la nota de corte, o de la oferta de plazas de una determinada titulación (es una carrera fácil, podía tener plaza, etc.).

Otra perspectiva con la que podemos enfocar las motivaciones para la elección de la carrera se centra en el estudio de las metas personales que los estudiantes tienen, respecto a las expectativas que ésta les ofrece. En este sentido, si nos centramos en el contenido de estas metas, Ford (1992) distingue entre:

- Metas que implican asertividad. El alumnado busca definirse como individuo al seleccionar la carrera, poder disponer de recursos económicos y ser valorado por sus iguales (los salarios en el sector son muy atractivos, hay una gran oferta de empleo, etc.).

- Metas de integración en el grupo. Búsqueda de amigos, sensación de pertenencia, justicia, equidad (tener la posibilidad de viajar y conocer gente, conocer nuevas culturas y gente de todo el mundo, etc.).

- Metas relacionadas con la tarea. Tener conocimientos sobre la materia propia de la titulación, ser creativo, experto en el área de estudio (permite crear su propia empresa, permite ser dinámico y creativo, etc.)

En el plano empírico podemos encontrar trabajos como el de Briggs (2006) dofnde se analizan los factores que influencian la elección de carrera universitaria con estudiantes de 6 universidades de Escocia, con una muestra de 651 estudiantes, centrándose en las titulaciones de contabilidad e ingeniería. La decisión es compleja e influenciada por una multitud de factores. Identifica variación en función de la universidad, la disciplina o el género, aunque encuentra que los factores más importantes son la reputación académica, la distancia del hogar y la localización.

Por su parte, Skatova y Ferguson (2014) identifican 4 razones para elegir carrera universitaria: aspectos de la propia titulación, interés intrínseco en la materia, oportunidad para ayudar a otros y opción sencilla de acceder a la educación superior. Estudian la elección en dos muestras de estudiantes (de 989 y 896 estudiantes) en universidades de Reino Unido mediante un cuestionario, encontrando diferentes razones predominantes según fueran carreras de Medicina, Ingenierías o Artes y Humanidades. En Artes y Humanidades los motivos son el interés en la materia, baja preocupación por la carrera profesional futura y opción sencilla de acceder a la educación superior. Encontraron también diferencias por género. Mientras que las mujeres se guiaban por ayudar a otros y el interés en la materia, los hombres se inclinaban más por la propia titulación y la opción sencilla de acceder a la educación superior.

En el ámbito universitario español, Ariño et al (2008) analizan una muestra de estudiantes de 4 universidades españolas, con 60 estudiantes entrevistados y 58 estudiantes participantes en grupos de discusión. Describen que los estudiantes tienen dos planteamientos en la decisión de elegir carrera universitaria: la vocación o la obligación. En el primer planteamiento vocacional, la elección de la carrera universitaria es aquella que se ajusta en mayor medida a la identidad personal, siendo más minoritario. El segundo planteamiento, más numeroso, se centra en la ética de la responsabilidad, qué carrera 
conviene elegir, debido al atractivo de una determinada profesión para la que forma o al interés laboral práctico relacionado con salidas profesionales, demanda por parte del mundo laboral o niveles salariales. Sin embargo, ambos planteamientos se ven influenciados en gran medida por la familia, considerando menor por ejemplo la influencia de servicios de orientación de los institutos. Peró et al. (2015) consideran que hay factores generales y subjetivos que los alumnos tienen en cuenta a la hora de elegir titulación en la universidad, realizando un estudio mediante un cuestionario que recoge 6 factores: consideración de la universidad, utilidad percibida, impacto social, aspectos vocacionales, influencia del entorno y localización geográfica. Analizan una muestra de 1532 estudiantes de 8 universidades españolas. Encuentran que las variables de género y área académica son importantes a la hora de identificar distintos perfiles.

En este trabajo se propone un enfoque apoyado en las expectativas del alumnado de nuevo ingreso en las titulaciones de Turismo con el objetivo de encontrar los motivos que impulsaron a los estudiantes de las titulaciones de Turismo y TADE a seleccionar estas carreras, incluyendo sus expectativas de empleo futuro. Consideramos que, al margen de otros elementos que pueden tener una influencia en el comportamiento del individuo y que aquí se han analizado, las expectativas del alumnado es un factor relevante a tener en cuenta, ya que orientan su comportamiento futuro en sus estudios. El interés, la predisposición, la implicación del alumnado en el centro universitario, la cantidad y calidad del tiempo dedicado al estudio, la relación con compañeros o la autoconfianza tienen una relación importante con las expectativas que le llevaron a la elección del título, e incluso a continuar con sus estudios o al abandono de los mismos. Conocer estas expectativas es de suma importancia para la Universidad, en la medida que, si estas expectativas no coinciden con las percepciones del alumnado, el porcentaje de fracasos académicos y abandonos puede ser significativo.

\section{METODOLOGÍA}

\subsection{Participantes e Instrumentos}

La encuesta se realizó a todos los estudiantes de la Universitat de València matriculados en primer curso en el Grado en Turismo y el Doble Grado en Turismo y ADE (TADE) en el curso 2017-2018. La muestra es por tanto de tipo no probabilístico, y la razón de centrarse en alumnos de primer curso se debe a que consideramos que tienen más recientes las variables que les llevaron a elegir esa titulación y están influenciados en menor medida por los contenidos y experiencias con los que ya cuentan los alumnos de segundo curso o superior.

El cuestionario creado para este trabajo constaba de 15 preguntas y se realizó online mediante el programa libre LimeSurvey entre el 12 y el 21 de noviembre de 2017 . Se realizó de forma anónima y se obtuvieron 170 respuestas de un total de 201 alumnos matriculados, de las cuales 56 pertenecen a TADE y 114 a Turismo.

El cuestionario recogía información diversa. Por una parte, un bloque de preguntas relacionadas con variables de tipo sociodemográfico (edad, sexo, nacionalidad), variables relacionadas con los estudios de grado cursados por el alumnado (Turismo o TADE), la 
nota de acceso al Grado, así como la vía de acceso (Bachillerato, Ciclo Formativo, Acceso Mayores de 25 años), y si había seleccionado esta titulación en primera opción o no. Por otra parte, otro bloque incluía las variables relacionadas con los intereses y los puestos de trabajo que, según sus expectativas consideraban más probables conseguir al finalizar sus estudios.

Las respuestas se puntuaron de acuerdo a una escala tipo Likert de 10 puntos, desde 1 (no se corresponde en absoluto) hasta 10 (se corresponde totalmente). Las preguntas se plantearon de forma cerrada para facilitar el tratamiento posterior de los datos, incluyendo un total de 17 variables de interés, que de forma amplia recogen los atributos más atractivos relacionados con el Sector Turístico y la Titulación y 25 puestos de trabajo de amplio contenido relacionados con las salidas profesionales identificadas en el Libro Blanco y asociados a los programas formativos de prácticas externas del Grado en Turismo. Los datos fueron analizados con el programa SPSS, versión 22.

La consistencia del cuestionario fue validada calculando el coeficiente Alfa de Cronbach para cada bloque de preguntas y obteniendo los resultados que muestra la Tabla 1:

Tabla 1

VALIDACIÓN DEL CUESTIONARIO REALIZADO

\begin{tabular}{|c|c|}
\hline & Estadísticas de fiabilidad \\
\hline Bloque de Ítems & Alfa de Cronbach \\
\hline Intereses &, 752 \\
\hline Salidas profesionales &, 925 \\
\hline
\end{tabular}

Como criterio general, George y Mallery (2003) sugieren que los coeficientes alfa superiores a 0,7 pueden ser considerados como aceptables, siendo los de mayor de 0,9 como excelentes. Por lo tanto, con los resultados obtenidos podemos asegurar la consistencia interna de los ítems analizados para cada uno de los bloques de preguntas.

\subsection{Análisis de datos}

En primer lugar, se calcularon los estadísticos descriptivos de todas las variables objeto de estudio (medias, desviaciones típicas y porcentajes). La media de edad de acceso a la titulación es de 18,7 años, siendo en Turismo ligeramente superior. Como podemos ver en la Tabla 2, en TADE todo el alumnado accede por Bachillerato, mientras que en Turismo el $7 \%$ accede por Ciclo Formativo y el 1,8\% por acceso de mayores de 25 años, siendo una mayoría el alumnado que accede por Bachillerato. En TADE solo hay el 3,6\% de nacionalidad no española, mientras que en Turismo hay un 20,2\% de estudiantes de nacionalidad no española, de los cuales el $30 \%$ de estos proceden de China. 


\section{Tabla 2 \\ ANÁLISIS DESCRIPTIVO DE LA MUESTRA}

\begin{tabular}{llcc}
\hline & & \multicolumn{2}{c}{ Grado } \\
\cline { 2 - 3 } & & Turismo & TADE \\
\hline $\begin{array}{l}\text { Edad (en años) Media } \\
\text { (Desviación típica) }\end{array}$ & 19,08 & 17,95 \\
\hline \multicolumn{2}{l}{$\begin{array}{l}\text { Nota acceso Universidad (sobre 14 puntos) Media } \\
\text { (Desviación típica) }\end{array}$} & $(2,2)$ & $(0,35)$ \\
\hline Acceso a la & Bachillerato & 8,83 & 11,32 \\
titulación & Ciclo Formativo & $(1,1)$ & $(0,6)$ \\
\hline & Acceso mayores de 25 años & $91,2 \%$ & $100 \%$ \\
\hline Nacionalidad & España & $7 \%$ & $0 \%$ \\
& Otras & $1,8 \%$ & $0 \%$ \\
\hline Sexo & Hombre & $79,8 \%$ & $96,4 \%$ \\
\hline Opción elección & Mujer & $20,2 \%$ & $3,6 \%$ \\
\hline titulación & 2 & $33,3 \%$ & $28,6 \%$ \\
& 3 & $66,7 \%$ & $71,4 \%$ \\
\hline Total respuestas & $\mathrm{N}$ & $66,7 \%$ & $76,8 \%$ \\
\hline
\end{tabular}

Podemos observar también en la Tabla 2 que hay una mayoría de mujeres en ambas titulaciones. La nota de acceso a la universidad también presenta diferencias, ya que la nota media en TADE es de 11,32 (sobre 14 puntos), mientras que la nota en Turismo es claramente menor, ya que es de 8,83 . Respecto a si eligieron esa titulación como primera, segunda, tercera o cuarta opción, también existen diferencias entre TADE y Turismo. En TADE el 76,8\% la elige como primera opción, y nadie como tercera o cuarta opción. En Turismo el porcentaje de primera opción disminuye al $66,7 \%$, y aparece un $12,3 \%$ de forma agregada que la eligen en tercera o cuarta opción.

Inicialmente se realizó una comparación de medias para comparar los perfiles de Turismo y TADE e identificar si el alumnado de Turismo y TADE había otorgado una puntuación significativamente diferente en los ítems analizados.

Posteriormente, se realizó un análisis factorial para analizar la información relacionada con los intereses que impulsan al alumnado en la elección de los estudios de Grado analizados. El análisis factorial o de componentes principales tiene como finalidad reducir la dimensión de las variables y detectar los factores de variabilidad más importantes (Hair et al., 1999). Esta técnica nos permite condensar la información original en unas nuevas variables sintéticas llamadas componentes o factores. Se trata de hacer los datos más inteligibles, para poder comprender la estructura y las interrelaciones existentes en 
el fenómeno que se estudia. Así, el análisis de componentes principales ha permitido resumir la información disponible sobre los 17 elementos de interés que han llevado a los estudiantes encuestados a elegir el grado de TADE y Turismo, suprimiendo redundancias y detectando las dimensiones más relevantes. Posteriormente se realizó un agrupamiento de medias por factores para explicar los motivos que llevaron al alumnado a cursar las titulaciones analizadas.

Por otra parte, se ha realizado un análisis descriptivo (media y desviación típica) de las expectativas del alumnado respecto a las posibilidades que estos consideran para conseguir cada uno de los puestos de trabajo relacionados con las salidas profesionales de las titulaciones analizadas. Esta información nos permite conocer cuáles son las profesiones que el alumnado considera más alcanzables en función de sus posibilidades, expectativas y estudios cursados.

\section{RESULTADOS}

En primer lugar, se ha realizado un análisis de diferencia de medias, con el fin de identificar aquellos intereses a los que el alumnado de Turismo y TADE han otorgado una puntuación significativamente diferente. Se trata, en definitiva, de observar diferencias en los perfiles de los alumnos de Turismo y TADE en cuanto a los factores que hacen más atractiva la elección de cada titulación.

Tabla 3

PUNTUACIÓN MEDIA Y DESVIACIÓN TÍPICA DE LOS INTERESES PLANTEADOS PARA LOS ALUMNOS DE TURISMO Y TADE

\begin{tabular}{llccccccc}
\hline & $\begin{array}{l}\text { Turismo } \\
\text { Media } \\
\text { (Desv. } \\
\text { estand.) }\end{array}$ & $\begin{array}{l}\text { TADE } \\
\text { Media } \\
\text { (Desv. estand.) }\end{array}$ & $\begin{array}{l}\text { Prueba Levene: Se } \\
\text { asumen varianzas } \\
\text { iguales }\end{array}$ & $\begin{array}{l}\text { Prueba t para } \\
\text { igualdad de } \\
\text { medias Sig. } \\
\text { (bilateral) }\end{array}$ \\
$\begin{array}{l}\text { 1-Me ha gustado siempre el } \\
\text { turismo o trabajar en turismo }\end{array}$ & 6,98 & $(2,44)$ & 6,27 & $(2,42)$ & Sí & 0,796 & 0,073 & $*$ \\
\hline $\begin{array}{l}\text { 2-Tendré la posibilidad de viajar y } \\
\text { conocer gente }\end{array}$ & 8,29 & $(1,72)$ & 7,54 & $(2,33)$ & No & 0,008 & 0,034 & $* *$ \\
\hline $\begin{array}{l}\text { 3-Tengo un negocio o trabajo en el } \\
\text { sector }\end{array}$ & 2,93 & $(2,88)$ & 2,34 & $(2,24)$ & No & 0,014 & 0,145 \\
\hline $\begin{array}{l}\text { 4-Me cambié de carrera y opté por } \\
\text { Turismo/TADE }\end{array}$ & 2,07 & $(2,62)$ & 1,59 & $(2,02)$ & No & 0,023 & 0,189 \\
\hline $\begin{array}{l}\text { 5-Considero que es una carrera fácil } \\
\text { y me permitirá tener un título oficial }\end{array}$ & 3,93 & $(2,54)$ & 2,64 & $(1,99)$ & No & 0,044 & 0,000 & $* *$ \\
\hline 6-Podré aprender idiomas & 8,40 & $(1,53)$ & 7,50 & $(2,40)$ & No & 0,000 & 0,012 & $* *$ \\
\hline 7-Es un sector creciente & 8,48 & $(1,61)$ & 7,86 & $(1,53)$ & Sí & 0,702 & 0,017 & $* *$ \\
\hline $\begin{array}{l}\text { 8-Es una oportunidad para trabajar } \\
\text { en el extranjero }\end{array}$ & 8,37 & $(1,57)$ & 7,79 & $(2,25)$ & No & 0,022 & 0,086 & $*$ \\
\hline
\end{tabular}




\begin{tabular}{|c|c|c|c|c|c|c|c|c|}
\hline \multirow[b]{2}{*}{ 9-Hay una gran oferta de empleo } & \multicolumn{2}{|c|}{$\begin{array}{l}\text { Turismo } \\
\text { Media } \\
\text { (Desv. } \\
\text { estand.) }\end{array}$} & \multicolumn{2}{|c|}{$\begin{array}{l}\text { TADE } \\
\text { Media } \\
\text { (Desv. estand.) }\end{array}$} & \multicolumn{2}{|c|}{$\begin{array}{l}\text { Prueba Levene: Se } \\
\text { asumen varianzas } \\
\text { iguales } \\
\\
\\
\mathrm{Sig.} .\end{array}$} & \multicolumn{2}{|c|}{$\begin{array}{l}\text { Prueba t para } \\
\text { igualdad de } \\
\text { medias Sig. } \\
\text { (bilateral) }\end{array}$} \\
\hline & 7,16 & $(2,02)$ & 6,43 & $(2,40)$ & Sí & 0,081 & 0,039 & $* *$ \\
\hline $\begin{array}{l}\text { 10-Conoceré nuevas culturas y } \\
\text { gente de todo el mundo }\end{array}$ & 8,32 & $(1,55)$ & 7,52 & $(1,97)$ & No & 0,045 & 0,009 & $* *$ \\
\hline 11-Los salarios son muy atractivos & 5,78 & $(2,06)$ & 4,79 & $(2,31)$ & Sí & 0,281 & 0,005 & $* *$ \\
\hline $\begin{array}{l}\text { 12-Me permitirá ser dinámico/a y } \\
\text { creativo/a }\end{array}$ & 6,85 & $(1,87)$ & 6,32 & $(2,31)$ & Sí & 0,069 & 0,111 & \\
\hline 13-Podré crear mi propia empresa & 6,27 & $(2,73)$ & 6,80 & $(2,92)$ & Sí & 0,736 & 0,246 & \\
\hline $\begin{array}{l}\text { 14-Contribuiré al crecimiento de } \\
\text { mi país }\end{array}$ & 6,07 & $(2,57)$ & 5,68 & $(2,72)$ & Sí & 0,631 & 0,361 & \\
\hline $\begin{array}{l}\text { 15-No tenía nota para el grado que } \\
\text { quería cursar }\end{array}$ & 3,04 & $(3,14)$ & 2,68 & $(3,24)$ & Sí & 0,829 & 0,481 & \\
\hline 16-Es un grado con prestigio & 4,60 & $(2,34)$ & 5,20 & $(2,67)$ & Sí & 0,069 & 0,136 & \\
\hline $\begin{array}{l}\text { 17-El turismo es un sector } \\
\text { importante en la economía española }\end{array}$ & 8,46 & $(1,76)$ & 8,23 & $(2,08)$ & Sí & 0,240 & 0,446 & \\
\hline
\end{tabular}

$* \mathrm{p}<0,1 ; * * \mathrm{p}<0,05$

Como se observa en la Tabla 3 , encontramos diferencias de medias significativas para los intereses $1,2,5,6,7,8,9,10$ y 11, donde los alumnos de Turismo, otorgan en media una puntuación más alta que los alumnos de TADE. La excepción la encontramos en el interés 13 (Podré crear mi propia empresa) y 16 (Es un grado con prestigio), en las que los alumnos de TADE han dado una puntuación más elevada que los de Turismo. A pesar de que estas últimas diferencias no son significativas, son bastante coherentes, ya que a los alumnos de TADE se les presupone un mayor interés por la empresa y el emprendimiento que a los de Turismo. Además, dado que la nota de corte para entrar en TADE es mayor que la de Turismo, también es lógico pensar que los alumnos de TADE tienen más en consideración la variable prestigio a la hora de elegir sus estudios.

En líneas generales, por tanto, parece que los estudiantes de Turismo muestran más interés en media, por aspectos relacionados con la internacionalización de la actividad turística. Mientras que el perfil de TADE, tiende a dar más importancia en media (aunque esta diferencia no es significativa) a la hora de elegir los estudios, al interés por la creación de empresas y al prestigio del grado.

Con el fin de agrupar las distintas variables de interés que han llevado a los estudiantes a cursar el grado de Turismo o TADE, se ha realizado un análisis de componentes principales.

En primer lugar, para verificar si el análisis factorial ofrece una agrupación de variables válida se debe obtener el test Kaiser-Meyer-Olkin KMO. En el cálculo de este test intervienen los coeficientes de correlación de todas las variables. Por tanto, cuando el índice sea menor de 0,5 , debe entenderse que no existen relaciones entre variables explicadas a partir de otras, con lo que no procedería seguir con el análisis factorial (Hair J. et al., 1999). 


\section{Tabla 4 \\ ÍNDICE DE KAISER-MEYER-OLKIN (KMO) Y TEST DE BARTLET DEL ANÁLISIS FACTORIAL}

\begin{tabular}{llr} 
Medida de adecuación muestral de Kaiser-Meyer-Olkin (KMO) &, 756 \\
\hline & Chi-cuadrado aproximado & 788,080 \\
Prueba de esfericidad de Bartlett & Grados de libertad & 136 \\
\cline { 2 - 2 } & Significatividad &, 000 \\
\hline
\end{tabular}

Como muestra la Tabla 4, se ha obtenido una puntuación del KMO superior a 0,5 y el test de Bartlett's resulta significativo, por lo que realizamos el análisis factorial con el método de componentes principales. Para facilitar la interpretación de los factores, los hemos rotado con el criterio varimax, usando la normalización de Kaiser, obteniendo 6 factores de intereses (Tabla 5).

Tabla 5

ANÁLISIS DE COMPONENTES PRINCIPALES DE LOS INTERESES DE LOS ALUMNOS DE TURISMO Y TADE PARA MATRICULARSE EN SU RESPECTIVA TITULACIÓN

\begin{tabular}{|c|c|c|c|c|c|c|c|}
\hline \multirow{2}{*}{ Factores } & \multirow[t]{2}{*}{ Intereses } & \multicolumn{6}{|c|}{ Componentes } \\
\hline & & 1 & 2 & 3 & 4 & 5 & 6 \\
\hline \multirow[t]{4}{*}{$\begin{array}{l}\text { 1. Interés por la } \\
\text { internacionalización }\end{array}$} & $\begin{array}{l}\text { I2.Tendré la posibilidad de viajar y } \\
\text { conocer gente }\end{array}$ & ,842 & ,118 & ,016 & ,041 & ,171 & ,029 \\
\hline & I6.Podré aprender idiomas & ,777 & ,082 & ,086 & ,115 & 202 & ,095 \\
\hline & $\begin{array}{l}\text { I10.Conoceré nuevas culturas y gente } \\
\text { de todo el mundo }\end{array}$ & ,640 & ,446 & ,038 &, 061 & ,027 & ,217 \\
\hline & $\begin{array}{l}\text { I8.Es una oportunidad para trabajar } \\
\text { en el extranjero }\end{array}$ & ,466 & ,440 & ,013 &, 324 & ,099 & ,309 \\
\hline \multirow{2}{*}{$\begin{array}{l}\text { 2. Interés por las } \\
\text { expectativas de } \\
\text { empleo del sector } \\
\text { turístico }\end{array}$} & I9.Hay una gran oferta de empleo & ,078 & 849 & ,029 & 242 & ,006 & 079 \\
\hline & I11.Los salarios son muy atractivos & ,156 &, 774 & ,328 & ,026 & ,012 & ,206 \\
\hline \multirow{4}{*}{$\begin{array}{l}\text { 3. Interés por el } \\
\text { emprendimiento y el } \\
\text { autoempleo }\end{array}$} & I13.Podré crear mi propia empresa & 061 & ,076 & ,806 & ,114 & 119 & 148 \\
\hline & $\begin{array}{l}\text { I14.Contribuiré al crecimiento de mi } \\
\text { país }\end{array}$ & ,043 & ,284 &, 651 &, 333 & ,095 & ,015 \\
\hline & $\begin{array}{l}\text { I3.Tengo un negocio o trabajo en el } \\
\text { sector }\end{array}$ & , 175 & ,028 &, $\mathbf{5 0 0}$ & ,297 & , 187 & ,347 \\
\hline & $\begin{array}{l}\text { I12.Me permitirá ser dinámico/a y } \\
\text { creativo/a }\end{array}$ & ,390 &, 304 & ,427 & ,142 & ,028 & ,014 \\
\hline
\end{tabular}




\begin{tabular}{|c|c|c|c|c|c|c|c|}
\hline Factores & Intereses & \multicolumn{6}{|c|}{ Componentes } \\
\hline \multirow{3}{*}{$\begin{array}{l}\text { 4. Interés por la } \\
\text { importancia del } \\
\text { sector turístico en la } \\
\text { economía española }\end{array}$} & $\begin{array}{l}\text { I17.El turismo es un sector importante } \\
\text { en la economía española }\end{array}$ &, 015 & ,029 & ,202 & ,849 & ,071 & ,026 \\
\hline & I7.Es un sector creciente & ,364 & ,320 & ,044 & ,646 & ,108 & ,027 \\
\hline & I16.Es un grado con prestigio &, 044 & ,320 & ,387 & ,404 & ,066 & ,397 \\
\hline \multirow[t]{3}{*}{$\begin{array}{l}\text { 5. Interés por la } \\
\text { Titulación }\end{array}$} & $\begin{array}{l}\text { I15.No tenía nota para el grado que } \\
\text { quería cursar }\end{array}$ & 022 &, 052 & ,076 & ,099 & ,820 & 055 \\
\hline & $\begin{array}{l}\text { I5.Considero que es una carrera fácil } \\
\text { y me permitirá tener un título oficial }\end{array}$ & ,067 & ,353 &, 150 & ,196 &, 561 & , 425 \\
\hline & $\begin{array}{l}\text { I1.Me ha gustado siempre el turismo } \\
\text { o trabajar en turismo }\end{array}$ &, 513 & 052 &, 129 & 220 &, 522 & ,248 \\
\hline 6. Otros intereses & $\begin{array}{l}\text { I4.Me cambié de carrera y opté por } \\
\text { Turismo/TADE }\end{array}$ & ,004 & ,042 & ,047 & ,019 &, 045 & ,783 \\
\hline
\end{tabular}

Por la naturaleza de las variables que componen cada factor, hemos etiquetado los siguientes tipos de intereses generales que han llevado a los estudiantes a cursar el grado en Turismo o TADE:

1. Interés por la internacionalización. Es un factor que incluye el interés del alumnado por cursar un Grado en el que considera tiene la oportunidad de desarrollar actividades que le permiten acceder a entornos fuera de nuestras fronteras.

2. Interés por las expectativas de empleo del sector turístico. Este factor hace referencia al atractivo de las titulaciones respecto a su inserción en el mercado laboral.

3. Interés por el emprendimiento y el autoempleo. Incluye la visión emprendedora del alumnado y el grado en el que puede conseguir estos objetivos cursando la titulación.

4. Interés por la importancia del sector turístico en la economía española. El factor agrupa las variables que han podido condicionar la elección de los grados por parte del alumnado a partir de la imagen del sector en su entorno y sus posibilidades futuras.

5. Interés por la titulación. Recoge la prioridad en la elección de la titulación entre otras titulaciones posibles por parte del alumnado, y el nivel de vocación por el turismo.

6. Otros motivos. Incluye una variable residual.

La Tabla 6 agrupa las medias de las diferentes variables que componen cada uno de los factores identificados en la Tabla 11, así como su desviación típica para el alumnado de Turismo y TADE.

El factor que más peso ha tenido por parte del alumnado en la elección de los grados de Turismo y TADE se corresponde con la expectativa de cursar un título que le permita desarrollarse profesionalmente en un entorno internacional. De esta forma, esperan que el grado les permita interactuar con otras culturas, poder trabajar en el extranjero y poder comunicarse en situaciones reales en diferentes idiomas. Por lo tanto, tendrá una mayor 
disposición a involucrarse en contenidos que desarrollen actividades durante la carrera que le lleven a conseguir uno de los objetivos principales por los que decidió cursar estos títulos.

Por otra parte, perciben que España es un país con un alto potencial turístico donde el sector tiene un peso importante en la economía, y este es un segundo motivo por el que han considerado estas titulaciones, pensando en las posibilidades futuras que pueden tener en el entorno laboral. Sin embargo, contrasta esta percepción con sus bajas expectativas de empleo por cuenta ajena y la creación de negocios propios relacionados con el sector. Esta posición es acorde con lo que hemos comentado en este artículo respecto a la precariedad laboral y la imagen del sector que, pese a su importancia económica, sigue sin tener la imagen que le corresponde en base a su potencial y peso en España.

Respecto a la titulación, el alumnado no considera que sea una carrera fácil, y dada la baja valoración que le han otorgado a la variable "Me ha gustado siempre el turismo o trabajar en turismo", podemos entender que la elección del grado no ha venido condicionada por un factor vocacional.

El factor "Otros intereses" no tiene carácter explicativo y, como podemos ver en el cuadro, presenta un alto grado de variabilidad, por lo que su significatividad es nula.

Tabla 6

PUNTUACIONES MEDIAS DE LOS FACTORES DE ATRACCIÓN

\begin{tabular}{|l|ccc|ccc|}
\hline & \multicolumn{3}{|c|}{ TURISMO } & \multicolumn{3}{c|}{ TADE } \\
\hline FACTORES & Media & $\begin{array}{c}\text { Desviación } \\
\text { Estándar }\end{array}$ & $\begin{array}{c}\text { Coeficiente } \\
\text { Variación }\end{array}$ & Media & $\begin{array}{c}\text { Desviación } \\
\text { Estándar }\end{array}$ & $\begin{array}{c}\text { Coeficiente } \\
\text { Variación }\end{array}$ \\
Internacionalización & 8,35 & 0,05 & $1 \%$ & 7,59 & 0,11 & $2 \%$ \\
Importancia Sector & 7,18 & 1,82 & $25 \%$ & 7,1 & 1,34 & $19 \%$ \\
Expectativas Empleo & 6,47 & 0,69 & $10 \%$ & 5,61 & 0,82 & $15 \%$ \\
Emprendimiento & 5,53 & 1,52 & $27 \%$ & 5,29 & 1,74 & $33 \%$ \\
Titulación & 4,65 & 1,68 & $36 \%$ & 3,86 & 1,7 & $44 \%$ \\
Otros intereses & 2,07 & 2,62 & $126 \%$ & 1,59 & 2,02 & $127 \%$ \\
\hline
\end{tabular}

Por lo que respecta a las diferencias en cuanto a valoración del alumnado de Turismo y TADE para cada factor no hay diferencias en cuanto a su rango de preferencias; sí que podemos destacar unas puntuaciones ligeramente más altas en la titulación de Turismo que en la de TADE. Esto tiene cierta lógica considerando que la doble titulación de TADE incluye alumnado con un doble perfil de Administración de Empresas y Turismo, y que las preguntas se centraban exclusivamente en el Turismo.

Complementariamente, se ha realizado un análisis descriptivo de las expectativas de los alumnos respecto a conseguir determinados puestos de trabajo relacionados con el turismo, de un listado de 25 puestos de trabajo (Tabla 7) los estudiantes valoraron cuáles eran sus preferencias y expectativas respecto a conseguir cada uno de estos puestos en una escala Likert de 10 puntos, desde 1 (el puesto no se ajusta a mis expectativas) hasta 10 (el 
puesto se ajusta totalmente a mis expectativas). Las preferencias de los estudiantes se han ordenado en función de sus medias, tanto para la titulación de Turismo como la de TADE.

Tabla 7

EXPECTATIVAS DE CONSEGUIR UN PUESTO DE TRABAJO CONCRETO RELACIONADO CON EL TURISMO

\begin{tabular}{|c|c|c|c|c|c|c|}
\hline & \multicolumn{3}{|c|}{ TURISMO } & \multicolumn{3}{|c|}{ TADE } \\
\hline & Media & $\begin{array}{l}\text { Desviación } \\
\text { típica }\end{array}$ & $\begin{array}{c}\text { Orden de } \\
\text { Preferencia }\end{array}$ & Media & $\begin{array}{l}\text { Desviación } \\
\text { típica }\end{array}$ & $\begin{array}{c}\text { Orden de } \\
\text { preferencia }\end{array}$ \\
\hline $\begin{array}{l}\text { L1- Subdirección de } \\
\text { empresa turística }\end{array}$ & 7,23 & 2,09 & 1 & 8,04 & 1,72 & 1 \\
\hline $\begin{array}{l}\text { L2- Técnico de } \\
\text { Administración }\end{array}$ & 5,91 & 2,27 & 22 & 7,41 & 1,72 & 6 \\
\hline L3- Técnico Comercial & 6,57 & 2,12 & 16 & 6,79 & 1,83 & 17 \\
\hline $\begin{array}{l}\text { L4- Técnico en Relaciones } \\
\text { Públicas }\end{array}$ & 6,98 & 2,37 & 9 & 6,98 & 2,25 & 14 \\
\hline L5- Técnico en Producto & 6,38 & 2,05 & 19 & 6,70 & 1,99 & 20 \\
\hline L6- Técnico en Calidad & 6,46 & 2,12 & 18 & 6,79 & 2,25 & 18 \\
\hline $\begin{array}{l}\text { L7- Técnico en Recursos } \\
\text { Humanos }\end{array}$ & 6,58 & 2,28 & 15 & 6,96 & 2,16 & 15 \\
\hline $\begin{array}{l}\text { L8- Técnico en Atención } \\
\text { al cliente }\end{array}$ & 6,70 & 2,49 & 14 & 6,80 & 2,25 & 16 \\
\hline $\begin{array}{l}\text { L9- Técnico de } \\
\text { Contratación de Productos } \\
\text { turísticos }\end{array}$ & 7,47 & 1,85 & 4 & 7,34 & 1,83 & 7 \\
\hline $\begin{array}{l}\text { L10- Técnico de gestión } \\
\text { de una institución pública } \\
\text { de planificación y política } \\
\text { turística }\end{array}$ & 6,72 & 2,32 & 13 & 7,23 & 1,90 & 10 \\
\hline $\begin{array}{l}\text { L11- Técnico gestor de } \\
\text { una institución pública de } \\
\text { promoción o director de una } \\
\text { campaña de destino }\end{array}$ & 7,18 & 2,11 & 7 & 7,45 & 2,07 & 5 \\
\hline $\begin{array}{l}\text { L12- Responsable de un } \\
\text { programa de desarrollo } \\
\text { turístico }\end{array}$ & 7,45 & 2,04 & 5 & 7,21 & 2,21 & 11 \\
\hline $\begin{array}{l}\text { L13- Agente de Desarrollo } \\
\text { Turístico }\end{array}$ & 7,16 & 2,17 & 8 & 7,57 & 1,90 & 3 \\
\hline $\begin{array}{l}\text { L14- Director o técnico } \\
\text { de una institución } \\
\text { dinamizadora de producto }\end{array}$ & 6,19 & 2,23 & 20 & 7,00 & 2,01 & 13 \\
\hline $\begin{array}{l}\text { L15- Técnico de gestión de } \\
\text { instalaciones de ocio }\end{array}$ & 6,83 & 2,15 & 11 & 7,05 & 2,04 & 12 \\
\hline
\end{tabular}




\begin{tabular}{|l|c|c|c|c|c|c|}
\hline & Media & $\begin{array}{c}\text { Desviación } \\
\text { típica }\end{array}$ & $\begin{array}{c}\text { Orden de } \\
\text { Preferencia }\end{array}$ & Media & $\begin{array}{c}\text { Desviación } \\
\text { típica }\end{array}$ & $\begin{array}{c}\text { Orden de } \\
\text { preferencia }\end{array}$ \\
\hline $\begin{array}{l}\text { L16- Técnico de gestión de } \\
\text { espacios culturales }\end{array}$ & 6,92 & 2,07 & 10 & 6,68 & 2,27 & 21 \\
\hline $\begin{array}{l}\text { L17- Técnico de gestión de } \\
\text { eventos }\end{array}$ & 7,82 & 2,01 & 2 & 7,30 & 2,20 & 8 \\
\hline $\begin{array}{l}\text { L18- Técnico de gestión de } \\
\text { instalaciones deportivas }\end{array}$ & 5,94 & 2,80 & 21 & 6,14 & 2,47 & 22 \\
\hline $\begin{array}{l}\text { L19- Técnico de } \\
\text { intermediación turística }\end{array}$ & 6,80 & 2,10 & 12 & 7,29 & 2,05 & 9 \\
\hline $\begin{array}{l}\text { L20- Técnico de } \\
\text { comercialización y } \\
\text { promoción de MICE } \\
\text { (Meetings, Incentivos, } \\
\text { Congresos y Eventos) }\end{array}$ & 7,54 & 2,17 & 3 & 7,52 & 1,91 & 4 \\
\hline $\begin{array}{l}\text { L21- Técnico de producto } \\
\text { MICE }\end{array}$ & 6,55 & 2,17 & 17 & 6,79 & 2,15 & 19 \\
\hline $\begin{array}{l}\text { L22- Jefe de oficina de } \\
\text { alquiler de automóviles }\end{array}$ & 4,81 & 2,75 & 24 & 4,75 & 2,62 & 25 \\
\hline L23- Consultor & 5,55 & 2,41 & 23 & 5,73 & 2,42 & 24 \\
\hline $\begin{array}{l}\text { L24- Director/a de mi } \\
\text { propia empresa turística }\end{array}$ & 7,25 & 2,81 & 6 & 8,30 & 2,13 & 2 \\
\hline L25- Profesor/a & 4,77 & 3,18 & 25 & 5,93 & 3,20 & 23 \\
\hline
\end{tabular}

Turismo N=114, TADE N=56

Para la titulación de Turismo, los cinco empleos con mayor expectativa fueron: Subdirección de empresa turística, Técnico de gestión de eventos, Técnico de comercialización y promoción MICE y Técnico de Contratación de productos turísticos y Responsable de un programa de desarrollo turístico; que corresponden a perfiles totalmente orientados a la naturaleza de la titulación. Mientras que en TADE fueron: Subdirección de empresa turística, Director/a de mi propia empresa turística, Agente de desarrollo turístico, Técnico de comercialización y promoción MICE y Técnico gestor de una institución pública de promoción o director de una campaña de destino. Aunque dos de los puestos con mayores expectativas coinciden, sí que se observa una clara orientación de los estudiantes de TADE hacia la gestión y administración de empresas, lo que parece lógico por su preparación en la titulación de ADE; no obstante, también es remarcable que los titulados en TADE desarrollen expectativas más ambiciosas y emprendedoras como se denota en su elección de puestos relacionados con la dirección y el emprendimiento.

Por lo que se refiere a los puestos menos valorados en la titulación de Turismo, estos fueron: Técnico de gestión de instalaciones deportivas, Técnico de Administración, Consultor, Jefe de Oficina de alquiler de automóviles y Profesor/a. Para la titulación de TADE: Técnico de gestión de espacios culturales, Técnico de gestión de instalaciones 
deportivas, Profesor/a, Consultor y Jefe de Oficina de alquiler de automóviles. En este caso ambas titulaciones son prácticamente coincidentes en las profesiones que les resultan menos atractivas.

\section{CONCLUSIONES}

El turismo es uno de los pilares básicos de la economía nacional en España, tanto por su contribución al PIB como al empleo. Sin embargo, pese a su importancia, sigue siendo un sector donde el valor del factor humano no está convenientemente reconocido. Posiblemente la diversidad del sector, junto a la precariedad laboral de los servicios han contribuido a esta situación. En este contexto, las universidades tienen un importante reto que supone formar a profesionales que contribuyan a profesionalizar el sector a la vez que dotarles de las competencias adecuadas para atender la realidad del entorno en el que se mueven las diferentes organizaciones relacionadas con el Turismo.

El estudio de los motivos que impulsan a una persona a estudiar una u otra carrera universitaria es un tema de sumo interés, no solo para las universidades sino también para los poderes públicos que deben implementar políticas públicas dirigidas a la distribución de recursos; además es un aspecto social importante en cuanto que si la elección no está condicionada por la demanda laboral se puede producir un gap entre la oferta de profesionales y la demanda de los mismos en el mercado de trabajo.

Sin embargo, aunque el estudio de las motivaciones que llevan a los estudiantes a elegir una u otra carrera ha sido analizado suficientemente desde distintos enfoques, es importante considerar que el éxito o fracaso académico, así como el futuro laboral, dependerá en gran medida de que las expectativas que el estudiante había puesto en su carrera se correspondan con la realidad que se encuentra en el grado universitario seleccionado; trasladándose esta visión incluso hacia su actitud frente al mercado laboral. Este enfoque no ha sido abordado anteriormente en la literatura especializada.

Por todas las razones argumentadas en el párrafo anterior, es importante conocer cuáles son las expectativas que han condicionado la elección de una carrera universitaria, pues el comportamiento del alumnado estará condicionado por estas. Por otra parte, las universidades deben estar atentas a estas expectativas y adaptar sus planes de estudio, considerando la información que estas aportan y las demandas propias del mercado laboral.

En turismo el interés por conocer las expectativas del alumnado cobra mayor importancia si cabe, dado que el sector actualmente no favorece el prestigio profesional y deben ser los propios universitarios que se incorporan al mercado de trabajo quienes tienen la misión de contribuir, no solo con sus conocimientos y habilidades, sino también con su motivación y vocación, a prestigiar el sector y dotarle de una mayor eficacia y eficiencia; aspecto que solo se puede conseguir si estos se incorporan al mercado laboral con sus expectativas cumplidas.

En este trabajo nuestro principal objetivo se ha centrado en conocer las expectativas que llevan al alumnado de nueva incorporación en las titulaciones relacionadas con turismo (Turismo y TADE) a cursar estos estudios en la Universitat de València. Por otra parte, se quería conocer cuáles son sus expectativas respecto a los puestos de trabajo que consideran más atractivos y para los que se ven más capacitados. 
Los factores que fueron identificados y que se corresponden con las expectativas del alumnado con los estudios de turismo fueron: Internacionalización, Expectativas de empleo, Emprendimiento, Importancia del sector e Interés por la titulación. Lo primero que espera un estudiante de turismo cuando selecciona estos estudios es que le capaciten para disponer de los conocimientos y habilidades que le permitan interactuar con nuevos entornos y culturas fuera de sus fronteras. En este sentido el grado es visto como una oportunidad para poder desarrollarse profesionalmente con una visión global y abierta. El alumnado es consciente de la importancia del sector en la economía española y su amplia oferta, por lo que espera que no exista mucha dificultad en conseguir un puesto de trabajo una vez finalizados sus estudios. Sin embargo, le otorga poca importancia a que la titulación le permita desarrollar una conducta más emprendedora, dinámica y creativa; al tiempo que no la considera una titulación con prestigio. Pensamos que estas bajas expectativas en emprendimiento y sentido de pertenencia por la titulación pueden estar relacionadas con la precariedad laboral que perciben dentro del sector, lo cual es un motivo importante de preocupación, tanto para la universidad como para el sector, dado que esto se va a trasladar de forma negativa a la motivación por la carrera y posiblemente a sus aspiraciones profesionales futuras; por tanto, revertir esta situación supone un reto importante.

Por lo que respecta a la comparativa entre las titulaciones de Turismo y TADE analizadas, no existen diferencias en cuanto a la jerarquización que hemos comentado en el párrafo anterior, solo ligeramente respecto a las puntuaciones medias otorgadas a cada uno de los factores de expectativas. Estos resultados nos pueden hacer pensar que la elección tanto de una como otra titulación vino condicionada por su relación con el Turismo, no con la Dirección de Empresas como podría ser el caso de TADE. Posiblemente la doble titulación es vista como un factor que puede incrementar sus posibilidades de empleo futuro o que el atractivo de la titulación de Turismo se ve reforzado por la unión con otro grado.

En cuanto a sus expectativas en los puestos de trabajo que esperan conseguir al finalizar la carrera, tanto el alumnado de Turismo como de TADE se decantan por perfiles profesionales con una conexión más directa con el sector; aunque en este caso sí podemos encontrar diferencias entre ambas titulaciones, puesto que el alumnado de TADE tiene una mayor preferencia hacia puestos relacionados con Administración y Gestión, con expectativas más ambiciosas para ocupar puestos relacionados con la dirección y la creación de empresas.

En referencia a las limitaciones del trabajo, somos conscientes de que las expectativas de los individuos están condicionadas por la visión que estos tienen de su entorno, por lo que sus argumentos suelen estar más relacionados con sus vivencias que por el conocimiento de la realidad. Esto puede explicar gran parte de sus respuestas e incluso no corresponder con la situación real; no obstante, pensamos que la realidad se construye a partir de las expectativas y que por extensión las conductas suelen estar guiadas por estas.

En este trabajo hemos identificado las expectativas del alumnado en cuanto a los grados relacionados con Turismo y que han determinado la elección de su carrera. No obstante, quedan por explicar los motivos por los que han dado esas respuestas y que formaría parte de un futuro estudio cualitativo en profundidad. Por otra parte, es de nuestro interés realizar un estudio de la misma naturaleza al alumnado de último año de carrera, con el objetivo de analizar las diferencias de expectativas al comienzo de sus estudios 
y a su finalización; lo que nos daría información sobre si su paso por la universidad ha supuesto o no un cambio de expectativas y si esto ha condicionado su conducta futura.

\section{BIBLIOGRAFÍA}

ALONSO, P y LOBATO, H. (2004): «Elementos que influyen en el fracaso universitario: un estudio descriptivo», REOP. Revista Española de Orientación y Psicopedagogía, vol. $16, \mathrm{n}^{\circ} 1$, pp. 63-79.

ANECA (2004): Libro Blanco del Título de Grado de Turismo. Madrid.

ARAUJO, N. y FRAIZ, J.A. (2013): «A formação turística na Espanha: evolução e oferta universitária atual», Caderno Virtual de Turismo, vol. 13, n 3, pp. 289-307.

ARIÑO, A.; HERNANDEZ, M.; LLOPIS, R.; NAVARRO, P. Y TEJERINA, B. (2008) El oficio de estudiar en la universidad: compromisos flexibles. Valencia, Publicaciones de la Universitat de València.

BOE (1963): Decreto 2427/1963, n 235, de 1 de octubre de 1963, pp. 14062-14063.

BOE (1987): Real Decreto 1497/1987, n 298, de 14 de diciembre de 1987, pp. 3663936643.

BOE (2015): Real Decreto 43/2015, no 29, de 3 de febrero de 2015, pp. 8088-8091.

BRIGGS, S. (2006): “An exploratory study of the factors influencing undergraduate student choice: the case of higher education in Scotland", Studies in Higher Education, vol. 31, no 6, pp. 705-722.

CHACÓN, O. (2004): Diseño, aplicación y evaluación de una propuesta de orientación vocacional para la educación media, diversificada y profesional venezolana. Tesis doctoral inédita. Tarragona, Universitat Rovira i Virgili.

FORD, M.E. (1992): Motivating humans: Goals, emotions and personal agency beliefs. Newbury Park, CA, Sage.

GÁMEZ, E. y MARRERO, H. (2003): «Metas y motivos en la elección de la carrera universitaria: un estudio comparativo entre psicología, derecho y biología», Anales de Psicología, vol. 19, nº 1, pp. 121-131.

GEORGE, D.; MALLERY, P. (2003): "Reliability analysis. SPSS for Windows, step by step: a simple guide and reference". Boston: Allyn \& Bacon, vol. 222, p. 232.

GUÀRDIA, J., PERÓ, M., HERVÁS, A., CAPILLA, R., SORIANO, P.P. y PORRAS, M. (2012): «Factores asociados con la decisión de cursar estudios universitarios de Psicología: Una aproximación mediante modelos de ecuaciones estructurales», Anuario de Psicología, Vol. 42, nº 1, pp. 85-102.

HAIR, J., ANDERSON, R.E., TATHAM, R.L. y BLACK, W.C. (1999): Análisis multivariante, $5^{\mathrm{a}}$ Edición. Madrid, Pearson, Prentice Hall.

HOFSTADT, R. y GÓMEZ, J.M. (2006): Competencias y Habilidades Profesionales para Universitarios. Madrid, Ediciones Díaz de Santos.

HOLLAND, J.L. (1985): Making vocational choices: A theory of vocational personalities and work environments. New Jersey, Prentice-Hall.

INE (2017): Cuenta Satélite del Turismo de España. Notas de prensa. Serie 2010-2016, Instituto Nacional de Estadística. 
INE (2017): Encuesta Frontur (Estadística de movimientos turísticos en frontera), Instituto Nacional de Estadística.

LENT, R., BROWN, S. y HACKETT, G. (1994): «Toward a Unifying Cognitive Theory of Career and Academic Interest, Choice, and Performance», Journal of Vocational Behavior, Vol. 45, pp. 79-122.

LILLO BAÑULS, A. (2009): «El papel del capital humano en el Sector Turístico: Algunas reflexiones y propuestas», Cuadernos de Turismo, no 24, pp. 53-64.

LOFQUIST, L.H. y DAWIS, R.V. (1969): Adjustment to work: A psychological view of counseling. New York, McGraw-Hill.

LÓPEZ, D. y NORZAGARAY, C. (2016): «Construcción y Validación de un Instrumento para evaluar factores disposicionales asociados a la elección de carrera», Enseñanza e Investigación en Psicología, n ${ }^{\circ} 2$, pp. 120-130.

MARISCAL, A. (2003): «La formación turística en Andalucía: input para la mejora de la calidad en el empleo turístico», Cuadernos de Turismo, n 12 , pp. 93-117.

MARRERO, J.R., RODRIGUEZ, P. y RAMOS, J.M. (2016): «La flexibilidad laboral en el sector turístico: Un análisis a partir de las ofertas de empleo», Estudios y Perspectivas en Turismo, vol. 25, $\mathrm{n}^{\mathrm{o}} 2$, pp. 143-163.

MASLOW, A. (1951): La Jerarquía de las necesidades humanas. México, Fondo de Cultura Económica.

McCLELLAND, D. (1989): Estudio de la motivación humana. Madrid, Narcea.

MORRIS, CH. y MAISTO, A. (2001): Introducción a la psicología. México, Pearson Educación.

OCDE (2001): Definition and Selection of Competencies: Theoretical and Conceptual Foundations.

OLIVÁN, C. (2014): Empleabilidad y empleo: formación universitaria y mercado de trabajo en España y Aragón. Zaragoza, Agencia de Calidad y Prospectiva Universitaria de Aragón.

PERÓ, M., SORIANO, P.P., CAPILlA, R., GUÀRDIA I OLMOS, J. y HERVÁS, A. (2015): Questionnaire for the assessment of factors related to university degree choice in Spanish public system: A psychometric study, Computers in Human Behavior, vol. 47, 128-138.

PORTER, S.R. Y UMBACH, P. (2006): «College Major Choice: An Analysis of PersonEnvironment Fit», Research in Higher Education, Vol. 47, no 4, pp. 429-449.

SANTROCK, J. (2002): Psicología de la Educación. México, McGraw-Hill.

SKATOVA, A. Y FERGUSON, E. (2014): "Why do different people choose different university degrees? Motivation and the choice of degree", Frontiers in Psychology, vol. 5, pp. 1-15.

SUÁREZ, B. (2014): «La Universidad española ante la empleabilidad de sus graduados: Estrategias para su mejora», REOP. Revista Española de Orientación y Psicopedagogía, vol. 25, nº 2, pp. 90-110.

UNIVERSITAT DE VALÈNCIA (2009): Memoria de Verificación del Título Oficial de Grado: Graduado/a en Turismo. Valencia. 
